\title{
On Generalized Knot Groups
}

\author{
Xiao-Song Lin* \\ Sam Nelson \\ xl@math.ucr.edu \\ knots@esotericka.org \\ Department of Mathematics, University of California, Riverside \\ 900 University Avenue, Riverside, CA, 92521
}

\begin{abstract}
Generalized knot groups $G_{n}(K)$ were introduced first by Wada and Kelly independently. The classical knot group is the first one $G_{1}(K)$ in this series of finitely presented groups. For each natural number $n, G_{1}(K)$ is a subgroup of $G_{n}(K)$ so the generalized knot groups can be thought of as extensions of the classical knot group. For the square knot $S K$ and the granny knot $G K$, we have an isomorphism $G_{1}(S K) \cong G_{1}(G K)$. From the presentations of $G_{n}(S K)$ and $G_{n}(G K)$, for $n>1$, it seems unlikely that $G_{n}(S K)$ and $G_{n}(G K)$ would be isomorphic to each other. We are able to show that for many finite groups $H$, the numbers of homomorphisms from $G_{n}(S K)$ and $G_{n}(G K)$ to $H$, respectively, are the same. Moreover, the numbers of conjugacy classes of homomorphisms from $G_{n}(S K)$ and $G_{n}(G K)$ to $H$, respectively, are also the same. It remains a challenge to us to show, as we would like to conjecture, that $G_{n}(S K)$ and $G_{n}(G K)$ are not isomorphic to each other for all $n>1$.
\end{abstract}

\section{Introduction}

Generalized knot groups were introduced by Wada [5] and Kelly [3] independently. Wada arrived at these group invariants of knots by searching for homomorphisms of the braid group $B_{n}$ into Aut $\left(F_{n}\right)$, while Kelley's work was related to knot quandles and Wirtinger-type presentations.

We find it convenient to introduce generalized knot groups through the language of quandles (see [1] and 2 ). A quandle is a set $Q$ with a binary operation $\triangleright: Q \times Q \rightarrow Q$ satisfying the conditions

(i) for all $x \in Q, x \triangleright x=x$,

(ii) for all $x, y \in Q$, there is a unique $z \in Q$ with $x=z \triangleright y$, and

(iii) for all $x, y, z \in Q,(x \triangleright y) \triangleright z=(x \triangleright z) \triangleright(y \triangleright z)$.

If $(Q, \triangleright)$ satisfies $(i i)$ and (iii) but not necessarily $(i)$, then $(Q, \triangleright)$ is a rack.

Quandles and knots are closely related; if we interpret quandle elements as arcs in a link diagram, then the three quandle axioms are just the three Reidemeister moves. Quandles and groups are also closely related; indeed, a group is a quandle with quandle operation given by conjugation.

More precisely, given a group $G$, there is a quandle $\operatorname{Conj}(G)$ with underlying set $G$ and quandle operation $\triangleright: G \times G \rightarrow G$ given by $x \triangleright y=y^{-1} x y$. Conversely, for any quandle $Q$ there is a group called the associated group of $Q, \operatorname{As}(Q)=F(Q) /\left\langle y^{-1} x y(x \triangleright y)^{-1} \forall x, y \in Q\right\rangle$, that is, the free group on $Q$ modulo the normal subgroup generated by relations obtained by setting $y^{-1} x y$ equal to $x \triangleright y$ for all elements of $Q \cdot \operatorname{As}(Q)$ is also called $\operatorname{Adconj}(Q)$, since the functor As : QUANDLES $\rightarrow$ GROUPS is the left adjoint to the functor Conj : QUANDLES $\rightarrow$ GROUPS.

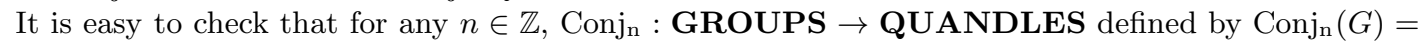
$\left(G, x \triangleright y=y^{-n} x y^{n}\right)$ is likewise a functor from the category of groups to quandles; its left adjoint, $\mathrm{As}_{\mathrm{n}}:$ QUANDLES $\rightarrow$ GROUPS given by $\operatorname{As}_{\mathrm{n}}(Q)=F(Q) /\left\langle x^{-n} y x^{n}(x \triangleright y)^{-1} \forall x, y \in Q\right\rangle$ is then a functor from quandles to groups. In particular, the fundamental group of a link complement is the associated group of the fundamental quandle of the link complement; if we now consider the $n$th associated group of the link's fundamental quandle, we obtain a new group invariant of links, which we will denote by $G_{n}(K)$ for a link $K$. Since $G_{1}(K)$ is just the usual fundamental group of $S^{3} \backslash K$, these groups form a family of generalized knot groups.

* Partially supported by NSF. 
It is well known that the knot group $G_{1}(K)$ alone is not strong enough to classify knots up to ambient isotopy. So, a natural question is whether the isomorphism types of generalized knot groups could be used as classifying invariants of knots. Unfortunately, this is not the case, as proposition [2.1] shows. However, we suspect that generalized knot groups hold additional information about knot type that is not present in the usual fundamental group.

As a testing case, we consider the square knot $S K$ and the granny knot $G K$. Then $G_{1}(S K)$ and $G_{1}(G K)$ are isomorphic to each other. To check whether $G_{n}(S K)$ and $G_{n}(G K)$ are isomorphic to each other, for $n \geq 2$, we programmed our computer to calculate the numbers of homomorphisms of $G_{n}(S K)$ and $G_{n}(G K)$ into a finite group $H$, respectively. To our surprise, for the many finite groups that we tested, these numbers are always equal to each other. We will show that this is indeed the case for any finite group satisfying a certain property. Furthermore, we will show that the numbers of conjugacy classes of homomorphisms of $G_{n}(S K)$ and $G_{n}(G K)$ into $H$, respectively, are also the same. Thus, we can not distinguish these two groups $G_{n}(S K)$ and $G_{n}(G K)$, for each $n \geq 2$, by simply counting (conjugacy classes of) homomorphisms into any finite group satisfying a certain property.

From the presentations of $G_{n}(S K)$ and $G_{n}(G K)$, it is very tempting to conjecture that $G_{n}$ distinguishes the square knot from the granny knot. If this is the case, then the groups $G_{n}$ contain additional information about knot type not contained in the fundamental group. Unfortunately, the situation turned out to be much more subtle than we originally thought. See the discussion in the last section about some computationally intensive attempts to show that the groups $G_{n}(S K)$ and $G_{n}(G K)$ are not isomorphic for $n \geq 2$. This conjecture remains open.

\section{The fundamental quandle and $G_{n}$}

Let $L$ be a link diagram. The fundamental quandle of $L$ has presentation

$$
\left.Q(L)=\left\langle x_{1}, \ldots, x_{m}\right| x_{i} \triangleright x_{j}=x_{k} \text { or } x_{k} \triangleright x_{j}=x_{i} \quad i=1, \ldots, m\right\rangle
$$

with one generator $x_{i}$ for each arc in $L$ and one relation for each crossing, either of the form $x \triangleright y=z$ where $x$ is the incoming underarc, $y$ is the overarc and $z$ is the outgoing overarc at a positive crossing, or $x \triangleright y=z$ where $x$ is the outgoing underarc, $y$ is the overarc and $z$ is the incoming overarc at a negative crossing.

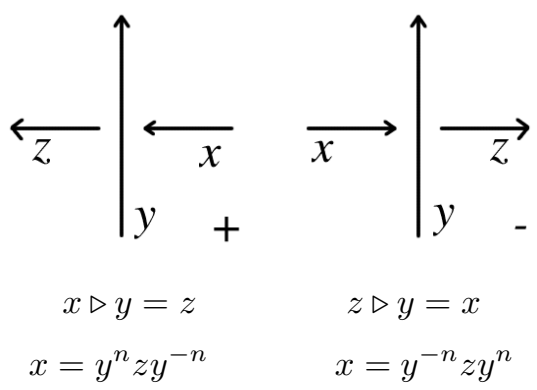

Figure 1: Quandle and $G_{n}$ relations at a crossing.

This then permits us to give a combinatorial description of $G_{n}(L)$ defined from a link diagram, namely $G_{n}(L)$ is the group with presentation

$$
\left.G_{n}(L)=\left\langle x_{1}, \ldots, x_{m}\right| x_{i}=x_{j}^{n} x_{k} x_{j}^{-n} \text { or } x_{i}=x_{j}^{-n} x_{k} x_{j}^{n}, i=1, \ldots, m\right\rangle,
$$

with the type of relation determined by the sign of the crossing.

Proposition 2.1 The right- and left-handed trefoils have isomorphic generalized groups $G_{n}$ for all $n \in$ $\mathbb{Z}_{+}$.

Proof. The right-hand trefoil has three positive crossings and generalized knot group

$$
G_{n}\left(T_{r}\right)=\left\langle a, b, c \mid a=b^{n} c b^{-n}, b=c^{n} a c^{-n}, c=a^{n} b a^{-n}\right\rangle .
$$


Using the fact that $\left(x^{n} y x^{-n}\right)^{m}=x^{n} y^{m} x^{-n}$, we can eliminate the generator $c$ to obtain

$$
\begin{aligned}
G_{n}\left(T_{r}\right) & =\left\langle a, b, \mid a=b^{n}\left(a^{n} b a^{-n}\right) b^{-n}, b=\left(a^{n} b a^{-n}\right)^{n} a\left(a^{n} b a^{-n}\right)^{-n}\right\rangle \\
& =\left\langle a, b, \mid a=b^{n}\left(a^{n} b a^{-n}\right) b^{-n}, b=\left(a^{n} b^{n} a^{-n}\right) a\left(a^{n} b^{-n} a^{-n}\right)\right\rangle \\
& =\left\langle a, b, \mid a=b^{n} a^{n} b a^{-n} b^{-n}, b=a^{n} b^{n} a b^{-n} a^{-n}\right\rangle \\
& =\left\langle a, b, \mid a b^{n} a^{n}=b^{n} a^{n} b, b a^{n} b^{n}=a^{n} b^{n} a\right\rangle .
\end{aligned}
$$

On the other hand, the left-handed trefoil has three negative crossings and generalized knot group

$$
G_{n}\left(T_{l}\right)=\left\langle a, b, c \mid a=b^{-n} c b^{n}, b=c^{-n} a c^{n}, c=a^{-n} b a^{n}\right\rangle .
$$

Thus,

$$
\begin{aligned}
G_{n}\left(T_{l}\right) & =\left\langle a, b, \mid a=b^{-n}\left(a^{-n} b a^{n}\right) b^{n}, b=\left(a^{-n} b a^{n}\right)^{-n} a\left(a^{-n} b a^{n}\right)^{n}\right\rangle \\
& =\left\langle a, b, \mid a=b^{-n}\left(a^{-n} b a^{n}\right) b^{n}, b=\left(a^{-n} b^{-n} a^{n}\right) a\left(a^{-n} b^{n} a^{n}\right)\right\rangle \\
& =\left\langle a, b, \mid a=b^{-n} a^{-n} b a^{n} b^{n}, b=a^{-n} b^{-n} a b^{n} a^{n}\right\rangle \\
& =\left\langle a, b, \mid b a^{n} b^{n}=a^{n} b^{n} a, a b^{n} a^{n}=b^{n} a^{n} b\right\rangle .
\end{aligned}
$$

So $G_{n}\left(T_{r}\right)$ and $G_{n}\left(T_{l}\right)$ are isomorphic for any $n$.

\section{$3 G_{n}$ of the square and granny knots}

The square knot $S K$ and the granny knot $G K$ are both connected sums of two trefoils; the granny knot is a connected sum of two right-handed trefoils or two left-handed trefoils, while the square knot is a connected sum of a right-handed trefoil and a left-handed trefoil.

It is well known that the fundamental groups of the complements of the square knot and the granny knot are isomorphic; therefore, the knot group and any knot invariants derived from it cannot distinguish these two knots. We will modify the defining presentations of $G_{n}(S K)$ and $G_{n}(G K)$ to a more symmetric form in this section.

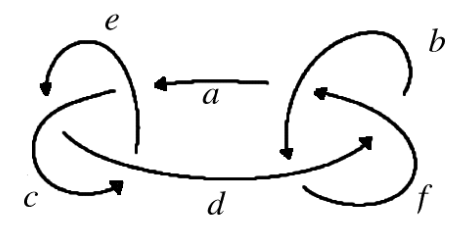

Figure 2: The square knot, SK

From the diagram in Figure 2 we obtain presentations of the fundamental quandle and $G_{n}$ of the square knot

$$
\begin{aligned}
Q(S K)= & \langle a, b, c, d, e, f| a \triangleright e=c, e \triangleright c=d, c \triangleright d=e, \\
& f \triangleright d=b, b \triangleright f=d, a \triangleright b=f\rangle, \\
G_{n}(S K)= & \langle a, b, c, d, e, f| a=e^{n} c e^{-n}, e=c^{n} d c^{-n}, \\
& \left.c=d^{n} e d^{-n}, f=d^{n} b d^{-n}, b=f^{n} d f^{-n}, a=b^{n} f b^{-n} \cdot\right\rangle
\end{aligned}
$$

Proposition $3.1 G_{n}(S K)$ has presentation

$$
\left\langle d, b, e \mid e d^{n} e^{n}=d^{n} e^{n} d, b d^{n} b^{n}=d^{n} b^{n} d, e^{n} d^{n} e d^{-n} e^{-n}=b^{n} d^{n} b d^{-n} b^{-n}\right\rangle .
$$

Proof. We reduce $G_{n}(S K)$ to the required form via a sequence of Tietze moves. First, we eliminate the generator $a$ :

$$
\begin{aligned}
G_{n}(S K)= & \langle c, b, d, e, f| b^{n} f b^{-n}=e^{n} c e^{-n}, e=c^{n} d c^{-n}, c=d^{n} e d^{-n}, \\
& \left.f=d^{n} b d^{-n}, b=f^{n} d f^{-n}\right\rangle .
\end{aligned}
$$


Next, we eliminate the generator $c$ :

$$
\begin{aligned}
G_{n}(S K)= & \langle b, d, e, f| b^{n} f b^{-n}=e^{n}\left(d^{n} e d^{-n}\right) e^{-n}, e=\left(d^{n} e^{n} d^{-n}\right) d\left(d^{n} e^{-n} d^{-n}\right), \\
& \left.f=d^{n} b d^{-n}, b=f^{n} d f^{-n}\right\rangle \\
= & \langle e, d, b, f| b^{n} f b^{-n}=e^{n} d^{n} e d^{-n} e^{-n}, e=d^{n} e^{n} d e^{-n} d^{-n}, \\
& \left.f=d^{n} b d^{-n}, b=f^{n} d f^{-n}\right\rangle .
\end{aligned}
$$

Next, we eliminate the generator $f$ :

$$
\begin{aligned}
G_{n}(S K)= & \langle b, d, e| b^{n}\left(d^{n} b d^{-n}\right) b^{-n}=e^{n} d^{n} e d^{-n} e^{-n}, e=d^{n} e^{n} d e^{-n} d^{-n}, \\
& \left.b=\left(d^{n} b^{n} d^{-n}\right) d\left(d^{n} b^{-n} d^{-n}\right)\right\rangle \\
= & \langle b, d, e| b^{n} d^{n} b d^{-n} b^{-n}=e^{n} d^{n} e d^{-n} e^{-n}, e=d^{n} e^{n} d e^{-n} d^{-n} \\
& \left.b=d^{n} b^{n} d b^{-n} d^{-n}\right\rangle .
\end{aligned}
$$

Rewriting slightly, we obtain

$$
\begin{aligned}
G_{n}(S K)= & \langle b, d, e| b d^{n} b^{n}=d^{n} b^{n} d, e d^{n} e^{n}=d^{n} e^{n} d, \\
& \left.b^{n} d^{n} b d^{-n} b^{-n}=e^{n} d^{n} e d^{-n} e^{-n}\right\rangle
\end{aligned}
$$

as required.

In the case $n=1$, the relation

$$
b^{n} d^{n} b d^{-n} b^{-n}=e^{n} d^{n} e d^{-n} e^{-n}
$$

becomes

$$
b d b d^{-1} b^{-1}=e d e d^{-1} e^{-1},
$$

which is a consequence of the other two relations, and we obtain

$$
G_{1}(S K)=\pi_{1}\left(S^{3} \backslash S K\right)=\langle b, d, e \mid d e d=e d e, d b d=b d b\rangle .
$$

Note that for $n>1$ we no longer have deficiency 1 .

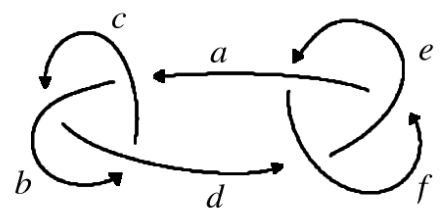

Figure 3: The granny knot, GK

From the diagram in Figure 3 we obtain presentations of the fundamental quandle and $G_{n}$ of the granny knot,

$$
\begin{aligned}
Q(G K)= & \langle a, b, c, d, e, f| a \triangleright c=b, c \triangleright b=d, b \triangleright d=c, d \triangleright f=e, \\
& f \triangleright e=a, e \triangleright a=f\rangle \\
G_{n}(G K)= & \langle a, b, c, d, e, f| a=c^{n} b c^{-n}, c=b^{n} d b^{-n}, b=d^{n} c d^{-n}, d=f^{n} e f^{-n}, \\
& \left.f=e^{n} a e^{-n}, e=a^{n} f a^{-n}\right\rangle .
\end{aligned}
$$

Proposition $3.2 G_{n}(G K)$ has presentation

$$
\begin{aligned}
G_{n}(G K)=\quad\langle d, b, e| b d^{n} b^{n}=d^{n} b^{n} d, d e^{n} d^{n}=e^{n} d^{n} e, \\
\left.e^{-n} d^{-n} e d^{n} e^{n}=b^{n} d^{n} b d^{-n} b^{-n} \cdot\right\rangle
\end{aligned}
$$


Proof. We reduce the presentation of $G_{n}(G K)$ obtained from the diagram. We begin by eliminating the generator $f$ :

$$
\begin{aligned}
G_{n}(G K)= & \langle a, b, c, d, e| a=c^{n} b c^{-n}, c=b^{n} d b^{-n}, b=d^{n} c d^{-n}, \\
& \left.d=\left(e^{n} a^{n} e^{-n}\right) e\left(e^{n} a^{-n} e^{-n}\right), e=a^{n}\left(e^{n} a e^{-n}\right) a^{-n}\right\rangle \\
= & \langle a, b, c, d, e| a=c^{n} b c^{-n}, c=b^{n} d b^{-n}, b=d^{n} c d^{-n}, \\
& \left.d=e^{n} a^{n} e a^{-n} e^{-n}, e a^{n} e^{n}=a^{n} e^{n} a\right\rangle \\
= & \langle a, b, c, d, e| a=c^{n} b c^{-n}, c=b^{n} d b^{-n}, b=d^{n} c d^{-n}, \\
& \left.d e^{n} a^{n}=e^{n} a^{n} e, e a^{n} e^{n}=a^{n} e^{n} a\right\rangle .
\end{aligned}
$$

Next, eliminating the generator $c$ yields

$$
\begin{aligned}
G_{n}(G K)= & \langle a, b, d, e| a=\left(b^{n} d^{n} b^{-n}\right) b\left(b^{n} d^{-n} b^{-n}\right), \\
& \left.b=d^{n}\left(b^{n} d b^{-n}\right) d^{-n}, d e^{n} a^{n}=e^{n} a^{n} e, e a^{n} e^{n}=a^{n} e^{n} a\right\rangle \\
= & \langle a, b, d, e| a=b^{n} d^{n} b d^{-n} b^{-n}, b=d^{n} b^{n} d b^{-n} d^{-n}, \\
& \left.d e^{n} a^{n}=e^{n} a^{n} e, e a^{n} e^{n}=a^{n} e^{n} a\right\rangle \\
= & \langle a, b, d, e| a=b^{n} d^{n} b d^{-n} b^{-n}, b d^{n} b^{n}=d^{n} b^{n} d, \\
& \left.d e^{n} a^{n}=e^{n} a^{n} e, e a^{n} e^{n}=a^{n} e^{n} a\right\rangle .
\end{aligned}
$$

Eliminating the generator $a$, using the fact that

$$
\left(b^{n} d^{n} b d^{-n} b^{-n}\right)^{m}=b^{n} d^{n} b^{m} d^{-n} b^{-n}
$$

yields

$$
\begin{aligned}
G_{n}(G K)= & \langle b, d, e| b d^{n} b^{n}=d^{n} b^{n} d, \\
& d e^{n}\left(b^{n} d^{n} b d^{-n} b^{-n}\right)^{n}=e^{n}\left(b^{n} d^{n} b d^{-n} b^{-n}\right)^{n} e, \\
& \left.e\left(b^{n} d^{n} b d^{-n} b^{-n}\right)^{n} e^{n}=\left(b^{n} d^{n} b d^{-n} b^{-n}\right)^{n} e^{n}\left(b^{n} d^{n} b d^{-n} b^{-n}\right)\right\rangle \\
= & \langle b, d, e| b d^{n} b^{n}=d^{n} b^{n} d, \\
& d e^{n}\left(b^{n} d^{n} b^{n} d^{-n} b^{-n}\right)=e^{n}\left(b^{n} d^{n} b^{n} d^{-n} b^{-n}\right) e, \\
& \left.e\left(b^{n} d^{n} b^{n} d^{-n} b^{-n}\right) e^{n}=\left(b^{n} d^{n} b^{n} d^{-n} b^{-n}\right) e^{n}\left(b^{n} d^{n} b d^{-n} b^{-n}\right)\right\rangle .
\end{aligned}
$$

The relation $b d^{n} b^{n}=d^{n} b^{n} d$ says that we can move $b$ past $d^{n} b^{n}$ to get $d$. In particular, we can apply this relation $n$ times to obtain $b^{n} d^{n} b^{n}=d^{n} b^{n} d^{n}$. Then

$$
\begin{aligned}
G_{n}(G K)= & \langle b, d, e| b d^{n} b^{n}=d^{n} b^{n} d, \\
& d e^{n}\left(b^{n} d^{n} b^{n}\right) d^{-n} b^{-n}=e^{n}\left(b^{n} d^{n} b^{n}\right) d^{-n} b^{-n} e, \\
& \left.e\left(b^{n} d^{n} b^{n}\right) d^{-n} b^{-n} e^{n}=\left(b^{n} d^{n} b^{n}\right) d^{-n} b^{-n} e^{n} b^{n} d^{n} b d^{-n} b^{-n}\right\rangle \\
= & \langle b, d, e| b d^{n} b^{n}=d^{n} b^{n} d, \\
& d e^{n}\left(d^{n} b^{n} d^{n}\right) d^{-n} b^{-n}=e^{n}\left(d^{n} b^{n} d^{n}\right) d^{-n} b^{-n} e, \\
& \left.e\left(d^{n} b^{n} d^{n}\right) d^{-n} b^{-n} e^{n}=\left(d^{n} b^{n} d^{n}\right) d^{-n} b^{-n} e^{n} b^{n} d^{n} b d^{-n} b^{-n}\right\rangle \\
= & \langle b, d, e| b d^{n} b^{n}=d^{n} b^{n} d, d e^{n} d^{n}=e^{n} d^{n} e, \\
& \left.e d^{n} e^{n}=d^{n} e^{n} b^{n} d^{n} b d^{-n} b^{-n}\right\rangle \\
= & \langle b, d, e| b d^{n} b^{n}=d^{n} b^{n} d, d e^{n} d^{n}=e^{n} d^{n} e, \\
& \left.e^{-n} d^{-n} e d^{n} e^{n}=b^{n} d^{n} b d^{-n} b^{-n}\right\rangle,
\end{aligned}
$$

as required.

As with the square knot, in the case $n=1$ the relation $e^{-n} d^{-n} e d^{n} e^{n}=b^{n} d^{n} b d^{-n} b^{-n}$ is a consequence of the other two relations, and we obtain

$$
G_{1}(G K)=\langle b, d, e \mid b d b=d b d, e d e=d e d\rangle \cong G_{1}(S K) .
$$

When $n>1$, we have

$$
\begin{aligned}
G_{n}(S K)= & \langle d, b, e| b d^{n} b^{n}=d^{n} b^{n} d, e d^{n} e^{n}=d^{n} e^{n} d \\
& \left.e^{n} d^{n} e d^{-n} e^{-n}=b^{n} d^{n} b d^{-n} b^{-n} \cdot\right\rangle
\end{aligned}
$$


and

$$
\begin{aligned}
G_{n}(G K)= & \langle d, b, e| b d^{n} b^{n}=d^{n} b^{n} d, d e^{n} d^{n}=e^{n} d^{n} e, \\
& \left.e^{-n} d^{-n} e d^{n} e^{n}=b^{n} d^{n} b d^{-n} b^{-n}\right\rangle .
\end{aligned}
$$

\section{Counting homomorphisms from $G_{n}(S K)$ and $G_{n}(G K)$ to a finite group}

We will use the presentations of $G_{n}(S K)$ and $G_{n}(G K)$ given in the end of the last section. Denote

$$
D=d^{n}, B=b^{n}, E=e^{n} \text {. }
$$

Then the subgroup of $G_{n}(S K)$ generated by $D, E, B$ is isomorphic to

$$
G_{1}(S K) \cong\langle D, B, E \mid B D B=D B D, E D E=D E D\rangle .
$$

That this subgroup is isomorphic to $G_{1}(S K)$ follows from the topological interpretation of $G_{n}$ given in [5]. We write the presentation of $G_{n}(S K)$ as follows:

$$
G_{n}(S K)=\left\langle d, b, e \mid b D B=D B d, e D E=D E d, E D e D^{-1} E^{-1}=B D b D^{-1} B^{-1}\right\rangle .
$$

If we plug the first and the second relations into the third relation, the later becomes

$$
E D D E d(E D D E)^{-1}=B D D B d(B D D B)^{-1} .
$$

Since $D=d^{n}$, we have $D d=d D$ and the third relation is equivalent to

$$
(E D)^{3} d(E D)^{-3}=(B D)^{3} d(B D)^{-3} .
$$

Let $H$ be a finite group. Homomorphisms of $G_{n}(S K)$ into $H$ may be constructed in the following way. Suppose first that we are given a homomorphism $\rho: G_{1}(S K) \longrightarrow H$. To define an extension $\hat{\rho}: G_{n}(S K) \longrightarrow H$, we first choose $\hat{\rho}(d) \in H$ such that $\hat{\rho}(d)^{n}=\rho(D)$. Then define

$$
\hat{\rho}(b)=\rho(D) \rho(B) \hat{\rho}(d) \rho(B)^{-1} \rho(D)^{-1} \quad \text { and } \quad \hat{\rho}(e)=\rho(D) \rho(E) \hat{\rho}(d) \rho(E)^{-1} \rho(D)^{-1} .
$$

Then if $\hat{\rho}$ also satisfies

$$
(\rho(E) \rho(D))^{3} \hat{\rho}(d)(\rho(E) \rho(D))^{-3}=(\rho(B) \rho(D))^{3} \hat{\rho}(d)(\rho(B) \rho(D))^{-3}
$$

for every choice of $\hat{\rho}(d) \in H$ such that $\hat{\rho}(d)^{n}=\rho(D)$, say that $H$ has property $T(n, S K)$. If $H$ satisfies property $T(n, S K)$ then every homomorphism from the subgroup into $H$ extends to a homomorphism from $G_{n}(S K)$ into $H$.

Example 4.1 If $H$ is abelian, then $H$ has property $T(n, S K)$ for every $n \in \mathbb{Z}$. If the exponent of $H$ is a multiple of $n$, then $D=B=E=1$, and $H$ has property $T(n, S K)$.

Contrary to our original conclusion, not every group satisfies property $T(n, S K)$; the map $\rho$ : $G_{1}(S K) \rightarrow S_{24}$ given by

$$
\begin{aligned}
& \rho(B)=(1,8,10,5,2,7,9,6)(15,17,24,19,16,18,23,20) \\
& \rho(D)=(3,5,12,7,4,6,11,8)(15,17,24,19,16,18,23,20) \\
& \rho(E)=(3,5,12,7,4,6,11,8)(13,20,22,17,14,19,21,18)
\end{aligned}
$$

with

$$
\hat{\rho}(d)=(3,15,5,17,12,24,7,19,4,16,6,18,11,23,8,20)
$$

satisfies $\hat{\rho}(d)^{2}=\rho(D), \rho(D) \rho(B) \rho(D)=\rho(B) \rho(D) \rho(B)$ and $\rho(D) \rho(E) \rho(D)=\rho(E) \rho(D) \rho(E)$ but fails to satisfy $(\rho(E) \rho(D))^{3} \hat{\rho}(d)(\rho(E) \rho(D))^{-3}=(\rho(B) \rho(D))^{3} \hat{\rho}(d)(\rho(B) \rho(D))^{-3}$. Hence $S_{24}$ does not satisfy property $T(n, S K)$. [4]

Proposition 4.1 Let $H$ be a finite group which satisfies property $T(n, S K)$. For $h \in H$, denote $\sqrt[n]{h}=$ $\left\{g \in H \mid g^{n}=h\right\}$. Then we have

$$
\left|\operatorname{Hom}\left(G_{n}(S K), H\right)\right|=\sum_{\rho \in \operatorname{Hom}\left(G_{1}(S K), H\right)}|\sqrt[n]{\rho(D)}| .
$$


For the group $G_{n}(G K)$, we have a similar presentation

$$
G_{n}(G K)=\left\langle d, b, e \mid b D B=D B d, d E D=E D e, E^{-1} D^{-1} e D E=B D b D^{-1} B^{-1}\right\rangle .
$$

Thus, we can define an analogous property $T(n, G K)$, and proposition 4.1 still holds if we replace $G_{n}(S K)$ by $G_{n}(G K)$. Therefore, we have the following conclusion.

Corollary 4.1 For any finite group $H$ satisfying both properties $T(n, S K)$ and $T(n, G k)$ we have

$$
\left|\operatorname{Hom}\left(G_{n}(S K), H\right)\right|=\left|\operatorname{Hom}\left(G_{n}(G K), H\right)\right|
$$

for each $n$.

Note that $H$ acts on $\operatorname{Hom}\left(G_{n}(S K), H\right)$ by conjugation. By our construction, two homomorphisms $\rho_{1}, \rho_{2} \in \operatorname{Hom}\left(G_{1}(S K), H\right)$ are conjugate to each other iff their extensions $\hat{\rho}_{1}, \hat{\rho}_{2} \in \operatorname{Hom}\left(G_{n}(S K), H\right)$ are conjugate to each other. So we have similar results like proposition 4.1 and corollary 4.1 for the numbers of conjugacy classes of $\operatorname{Hom}\left(G_{n}(S K), H\right)$ and $\operatorname{Hom}\left(G_{n}(G K), H\right)$, respectively, for each $n$.

\section{$5 \quad$ Are $G_{n}(S K)$ and $G_{n}(G K)$ isomorphic for $n \geq 2$ ?}

There is obviously a subtle difference in the presentations of $G_{n}(S K)$ and $G_{n}(G K)$ for $n \geq 2$. So even though we have not been able to distinguish these two groups via counting homomorphisms into finite groups, we think that these two groups should be not isomorphic to each other for each $n \geq 2$.

In [6], Wada defined the twisted Alexander polynomial for a finitely presented group $G$ associated with two homomorphisms from $G$ into a free abelian group of finite rank and a matrix group, respectively. For the groups $G_{n}(S K)$ and $G_{n}(G K)$, their abelianizations are both infinite cyclic. So we can have a twisted Alexander polynomial if we have a homomorphism into a matrix group. Using Maple, we calculated the twisted Alexander polynomials for $G_{n}(S K)$ and $G_{n}(G K)$ associated with homomorphisms into $S L(2, p)$ and $\operatorname{PSL}(2, p)$ for some small primes $p$ and small $n$. So far, we are not able to distinguish these two groups using twisted Alexander polynomials. For example, using the isomorphism $P S L(2,7) \cong S L(3,2)$, we calculated the total of 8232 twisted Alexander polynomials for both $G_{3}(S K)$ and $G_{3}(G K)$ associated with homomorphisms into $P S L(2,7)$. It turns out that these two sets of 8232 twisted Alexander polynomials are equal to each other.

We have also computed $\left|\operatorname{Hom}\left(G_{n}(S K), H\right)\right|$ and $\left|\operatorname{Hom}\left(G_{n}(G K), H\right)\right|$ for various values of $n$ for selected groups $H$ with orders as large as 360, always with the results that the two are equal. Computing $\left|\operatorname{Hom}\left(G_{n}(S K), S_{24}\right)\right|$ and $\left|\operatorname{Hom}\left(G_{n}(G K), S_{24}\right)\right|$ is presently beyond the capabilities of our hardware.

Despite such unsuccessful attempts to show that $G_{n}(S K)$ and $G_{n}(G K)$ are not isomorphic for $n \geq 2$, we still propose the following conjecture:

Conjecture $5.1 G_{n}(S K)$ and $G_{n}(G K)$ are not isomorphic for each $n \geq 2$.

\section{References}

[1] R. Fenn and C. Rourke, Racks and links in codimension two. J. Knot Theory Ramifications 1 (1992) 343-406.

[2] D. Joyce. A classifying invariant of knots, the knot quandle. J. Pure Appl. Algebra 23 (1982) 37-65.

[3] A. J. Kelly. Groups from Link Diagrams. Doctoral Thesis, U. Warwick (1990).

[4] C. Tuffley, private communication.

[5] M. Wada. Group invariants of links. Topology 31(1992) 399-406.

[6] M. Wada. Twisted Alexander polynomial for finitely presentable groups. Topology 34(1994) 241-256. 